\title{
Method of Group Decision Making with Interval Grey Numbers based on Grey Correlation and Relative Close Degree
}

\author{
Dan LIU, Bin HU*, Zhuo DING, Evangelos I. KAISARB
}

\begin{abstract}
With the development of society, big data's concept is mentioned more and more. However, in the numerous and complex mass data, the effective information is often very limited, and the data often show the complex characteristics of "close and orderly short-term correlation and large long-term disturbance", which is difficult to be expressed by a long sequence composed of a single real value. This paper takes the interval grey number as the research object for the uncertain system of "less data and poor information". According to the multi-attribute group decisions problem with attribute's values of interval grey number and the weights of decision-makers and attributes that are completely unknown, a method of group decision making with interval grey numbers based on grey correlation and relative close degree is proposed. For the purpose of making effective decisions, the weights of decision-makers and attributes are calculated by correlation degree of decision information which is measured by the model of grey correlation analysis based on interval grey number deviation degree. On this basis, the correlation degree between the scheme and the positive and negative ideal schemes are computed, and then the grey correlation and relative close degree of the scheme is calculated. Finally, the scheme is sorted according to the value of the grey correlation and relative close degree. The rationality and feasibility of the proposed method are verified by numerical examples.
\end{abstract}

Keywords: grey decision making; group decision making; interval grey number; weight analysis

\section{INTRODUCTION}

The problem of multi-attribute decision-making widely exists in various fields such as economic, management, engineering, military. For example, Ibrahim Badi \& Pamucar [1] proposed a method to implement a hybrid Grey theory-MARCOS method for decisionmaking regarding the selection of suppliers in the Libyan Iron and Steel Company (LISCO) to help it compete. Si et al. [2] propose a new method to calculate the score to rank the PFNs using positive ideal solution, negative ideal solution and average neutral value of the alternatives. Neutral degree of PFS has an active role in our proposal. We consider the average value of the neutral degree as a pivot point concerning the all other neutral degrees.

Under the background of the information age, due to the fact that massive information cannot directly reflect the real characteristics of the system, the effective information in the system is often very limited. Therefore, it is more and more difficult for single and long-term observations to accurately express the description of an object, and the research data is developing towards fuzziness and diversification. In view of the ambiguity and complexity of the data, the interval grey number in the grey system theory can be used to represent the uncertain number in an interval or a set of general numbers [3], which can effectively express the complex information that cannot be contained by a single real value. Different from the general interval number, the interval grey number is an uncertain number in which the interval range is known and the true value is unknown. The interval grey number cannot only cover the range of uncertain information, but also represent the data with uncertain grey level of the information contained in the interval. The upper and lower limits of the interval range represent the most conservative and optimistic views of the decision-maker, and the corresponding decisionmaking problem also shows some uncertainty, which prevents the decision-maker from making decisions in accordance with the deterministic method. Therefore, the research of uncertain decision-making has important theoretical significance and application value. Interval grey number, as a means to deal with multi-attribute uncertain decision-making problems, has attracted much attention from experts and scholars since the notion was introduced, and proposed multiple methods to solve uncertain multiattribute decision-making problems [4-12].

For the problem of multi-attribute group decisionmaking with interval grey number, there are relatively few studies. Among them, in the case of completely unknown attribute weights and expert weights, Chen \& Liu [13] established a group relevance scoring matrix by calculating the grey relevance between the decision information of each decision maker and the positive ideal scheme. On this basis, respectively based on the projected feature vector method and the fuzzy complementary judgment matrix ranking method rank the schemes. Yue et al. the advantage of this method is that the relative gap between the comprehensive relevance of the schemes obtained is relatively large. The smaller the correlation between the use of decision-making information, the more conducive to the attributes of evaluation, which promotes the scientific and effective decision-making. Yue [14] uses the projection method to determine the correlation between the decision-maker's decision vector and the group decision vector for the situation where the attribute weights are known and the expert weights are unknown. For the uncertain multi-attribute group decision-making problem with unknown attribute weights and expert weights, Yan et al. [15] established the planning model by using the grey correlation between each scheme and the ideal scheme and the principle of maximum entropy to find the attribute weight and expert weight. Based on the weighting method, the group decision result is obtained. For the uncertain multi-attribute group decision-making problem with unknown expert weights and partially known attribute weights, Li et al. [16] uses the grey correlation analysis method to obtain the correlation coefficients between the kernels and greys of the expert's kernel and the greyscale ideal scheme, Combine the two to get the expert weight, and further integrate the expert opinions through the weighting method. The common features of the above methods are: 1) When determining expert weights or 
attribute weights, they are achieved by calculating the correlation between decision information and positive ideal solutions (or group decisions); 2) When obtaining expert weights and attribute weights on the basis, the group evaluation value of the scheme is obtained through the weighting method.

We know that when the correlation between decisionmaking information is greater, the pros and cons between plans are difficult to characterize, and more unfavourable to evaluation; conversely, the smaller the correlation between decision-making information, the more favourable to evaluation. Therefore, this paper analyses from the perspective of "facilitating effective decisionmaking", and proposes an interval grey number group decision-making method based on the closeness of grey correlation. Finally, an example is used to verify the method proposed in the article, the feasibility and rationality of the method.

\section{METHOD}

Let $a(\otimes)=\left[a^{L}, a^{U}\right], b(\otimes)=\left[b^{L}, b^{U}\right]$ is set to the interval grey number, there is [14]:

1) $a(\otimes)=b(\otimes) \Leftrightarrow a^{L}=b^{L}$ and $a^{U}=b^{U}$;

2) $a(\otimes)+b(\otimes)=\left[a^{L}+b^{L}, a^{U}+b^{U}\right]$;

3) $k \cdot a(\otimes)=\left[k \cdot a^{L}, k \cdot a^{U}\right], k \geq 0$.

Let $\otimes_{1}=\left[\otimes_{1}^{L}, \otimes_{1}^{U}\right], \otimes_{2}=\left[\otimes_{2}^{L}, \otimes_{2}^{U}\right]$ is set to the interval grey number [11], remember $l\left(\otimes_{1}\right)=\otimes_{1}^{U}-\otimes_{1}^{L}, l\left(\otimes_{2}\right)=\otimes_{2}^{U}-\otimes_{2}^{L}$, and said

$d\left(\otimes_{1}, \otimes_{2}\right)=\frac{\left|\otimes_{1}^{L}-\otimes_{2}^{L}\right|+\left|\otimes_{1}^{U}-\otimes_{2}^{U}\right|}{l\left(\otimes_{1}\right)+l\left(\otimes_{2}\right)}$

is the degree of separation between the grey number.

Let the system behaviour sequence be

$$
\begin{gathered}
\otimes_{0}=\left[\otimes_{1}^{0}, \otimes_{2}^{0}, \cdots, \otimes_{n}^{0}\right] \\
\otimes_{1}=\left[\otimes_{1}^{1}, \otimes_{2}^{1}, \cdots, \otimes_{n}^{1}\right] \\
\vdots \\
\otimes_{m}=\left[\otimes_{1}^{m}, \otimes_{2}^{m}, \cdots, \otimes_{n}^{m}\right]
\end{gathered}
$$

where, $\otimes_{j}^{i}(i=1, \ldots, m ; j=1, \ldots, n)$ is the interval grey number.

Define the grey correlation [11] between $\otimes_{0}$ and $\otimes_{i}$ be

$$
R\left(\otimes_{0}, \otimes_{i}\right)=\sum_{j=1}^{n} w_{j} R\left(\otimes_{j}^{0}, \otimes_{j}^{i}\right)
$$

where $R\left(\otimes_{j}^{0}, \otimes_{j}^{i}\right)$ is the grey correlation coefficient of $R\left(\otimes_{0}, \otimes_{i}\right)$ at point $j$, which is defined as

$$
R\left(\otimes_{j}^{0}, \otimes_{j}^{i}\right)=\frac{\min _{i} \min _{j} d\left(\otimes_{j}^{0}, \otimes_{j}^{i}\right)+\xi \max _{i} \max _{j} d\left(\otimes_{j}^{0}, \otimes_{j}^{i}\right)}{d\left(\otimes_{j}^{0}, \otimes_{j}^{i}\right)+\xi \max _{i} \max _{j} d\left(\otimes_{j}^{0}, \otimes_{j}^{i}\right)}
$$

among them, $\xi \in(0,1)$ is called the resolution coefficient; function $d(\cdot)$ is shown in Eq. (1).

The grey relevance degree $R\left(\otimes_{0}, \otimes_{i}\right)$ defined by Eq. 3 satisfies the four axioms of grey relevance: normative, integrity, even symmetry and proximity [7].

\section{MULTI-ATTRIBUTE GROUP DECISION-MAKING 3.1 Description of Interval Grey Number Type Multi- Attribute Group Decision}

Let the expert group be $E=\left\{e_{1}, \ldots, e_{s}\right\}$ and the decision plan set be $X=\left\{x_{1}, \ldots, x_{m}\right\}$; each alternative plan has $n$ attributes, and the decision attribute set is $U=\left\{u_{1}, \ldots, u_{n}\right\}$. The evaluation matrix given by the decision maker $e_{k}$ is $\otimes_{k}=\left(\otimes_{i j}^{k}\right)_{m \times n}$, where $\otimes_{i j}^{k}$ represents the evaluation information of the decision maker $e_{k}$ about the attribute $u_{j}$ of the scheme $x_{i}$, the evaluation information is the interval grey number, and $\otimes_{i j}^{k L}, \otimes_{i j}^{k U}$ represents the lower limit and upper limit of the interval grey number $\otimes_{i j}^{k}$, respectively.

\subsection{Multi-Attribute Group Decision Analysis and Algorithm Method}

In the multi-attribute group decision-making problem, the key issue involved is the determination of attribute weights and expert weights. The usual determination method is to determine the expert weight by calculating the association between the individual expert and the expert group. However, the accuracy of the evaluation results of the expert group has yet to be verified. This article analyses the correlation between experts. When the correlation between a certain expert's opinion and other expert's opinions is greater, the advantages and disadvantages between the plans are difficult to describe, which is not conducive to evaluation; otherwise, it is conducive to evaluation. Similarly, the determination of attribute weights also has the above characteristics. Therefore, this paper adopts the idea favourable to evaluation" to determine attribute weights and expert weights respectively.

(1) The idea of determining the attribute weight: Under a certain attribute, take the vectors of each expert about all schemes as a sequence, use the grey correlation analysis method to calculate the correlation between the two sequences, and then use the cumulative grey correlation as the attribute Weights. Obviously, when the degree of association is greater, it is not conducive to evaluation, and the corresponding attribute weight should be smaller; otherwise, the corresponding attribute weight is greater.

(2) The way to determine the weight of experts: determine the weight of an expert by calculating the cumulative correlation between an expert and other experts. The greater the cumulative correlation, the smaller 
the corresponding expert weight assignment; otherwise, the corresponding expert weight assignment is greater.

Based on the above analysis, the following multiattribute group decision-making method based on grey closeness is established.

\subsection{Multi-Attribute Group Decision-Making Method Based on Grey Closeness}

Step 1 Normalization of decision matrix $\otimes_{k}(k=1,2$, $\ldots, s)$

There are two commonly used attributes of evaluation indicators: one is the benefit-type attribute, and the other is the cost-type attribute. In order to eliminate the influence of dimension and make each attribute comparable, the decision matrix $\otimes_{k}$ is normalized and still recorded as $\otimes_{k}$.

Step 2 Determine attribute weight, for attribute $u_{j}$, remember

$$
\Delta_{j}=\left[\begin{array}{cccc}
\otimes_{1 j}^{1} & \otimes_{2 j}^{1} & \cdots & \otimes_{m j}^{1} \\
\bigotimes_{1 j}^{2} & \bigotimes_{2 j}^{2} & \cdots & \otimes_{m j}^{2} \\
\vdots & \vdots & & \vdots \\
\otimes_{1 j}^{s} & \bigotimes_{2 j}^{s} & \cdots & \otimes_{m j}^{s}
\end{array}\right]=\left[\begin{array}{c}
\otimes_{j}^{1} \\
\otimes_{j}^{2} \\
\vdots \\
\otimes_{j}^{s}
\end{array}\right]
$$

Among them, the row vector $\otimes_{j}^{k}=\left[\begin{array}{llll}\otimes_{1 j}^{k} & \otimes_{2 j}^{k} & \cdots & \otimes_{m j}^{k}\end{array}\right](k=1, \ldots, s)$ is the expert $e_{k}$ gives the decision vector of each scheme under the index $u_{j}$.

Apply Eq. (4) to calculate the grey correlation between row vectors $\otimes_{j}^{l}, \otimes_{j}^{k}(l \neq k)$, which is

$$
R\left(\otimes_{j}^{l}, \otimes_{j}^{k}\right)=\frac{1}{m} \sum_{i=1}^{m} \frac{\min _{1 \leq i \leq m} d\left(\otimes_{i j}^{l}, \otimes_{i j}^{k}\right)+\xi \max _{1 \leq i \leq m} d\left(\otimes_{i j}^{l}, \otimes_{i j}^{k}\right)}{d\left(\otimes_{i j}^{l}, \bigotimes_{i j}^{k}\right)+\xi \max _{1 \leq i \leq m} d\left(\otimes_{i j}^{l}, \bigotimes_{i j}^{k}\right)}
$$

Therefore, for attribute $u_{j}$, the cumulative grey correlation is

$$
E_{j}=\sum_{k=1}^{s} \sum_{j=k+1}^{s} R\left(\otimes_{j}^{k}, \otimes_{j}^{l}\right)
$$

Define the attribute weight of index $u_{j}$ and the calculation formula of $w_{j}$ is as follows

$$
w_{j}=\frac{\left(\frac{1}{E_{j}}\right)}{\sum_{j}\left(\frac{1}{E_{j}}\right)}
$$

Step 3 Determine expert weights. First, give the definition of grey correlation between two decision matrices $\otimes_{k}, \otimes_{l}(k \neq l)$

$$
R\left(\otimes_{k}, \otimes_{l}\right)=\frac{1}{m \times n} \sum_{i=1}^{m} \sum_{j=1}^{n} R\left(\otimes_{i j}^{k}, \otimes_{i j}^{l}\right)
$$

and

$$
R\left(\otimes_{i j}^{k}, \otimes_{i j}^{l}\right)=\frac{\min _{i} \min _{j} d\left(\bigotimes_{i j}^{k}, \bigotimes_{i j}^{l}\right)+\xi \max _{i} \max _{j} d\left(\otimes_{i j}^{k}, \bigotimes_{i j}^{l}\right)}{d\left(\otimes_{i j}^{k}, \bigotimes_{i j}^{l}\right)+\xi \max _{i} \max _{j} d\left(\otimes_{i j}^{k}, \otimes_{i j}^{l}\right)}
$$

Expert $e_{k}$ has a cumulative relevance of $E_{k}=\sum_{l \neq k} R\left(\otimes_{k}, \otimes_{l}\right)$, and defines expert weight $\lambda_{k}$ as follows:

$$
\lambda_{k}=\frac{\frac{1}{E_{k}}}{\sum_{l=1}^{s} \frac{1}{E_{l}}}
$$

Step 4 Calculate the grey closeness of each scheme

1) Determine the positive ideal scheme $\otimes^{+}$and the negative ideal scheme $\otimes^{-}$, there are

$$
\begin{aligned}
& \otimes^{+}=\left(\otimes_{1}^{+}, \cdots, \otimes_{n}^{+}\right) \\
& \otimes^{-}=\left(\otimes_{1}^{-}, \cdots, \otimes_{n}^{-}\right)
\end{aligned}
$$

Among them,

$$
\begin{aligned}
& \otimes_{j}^{+}=\left[\otimes_{j}^{+L}, \otimes_{j}^{+U}\right]=\left[\max _{\mathrm{k}} \max _{\mathrm{i}} \otimes_{i j}^{k L}, \max _{\mathrm{k}} \max _{\mathrm{i}} \otimes_{i j}^{k U}\right] \\
& \otimes_{j}^{-}=\left[\otimes_{j}^{-L}, \otimes_{j}^{-U}\right]=\left[\min _{k} \min _{i} \otimes_{i j}^{k L}, \min _{k} \min _{i} \otimes_{i j}^{k U}\right]
\end{aligned}
$$

2) Combine the attribute weights to calculate the grey correlation degrees $R\left(\otimes^{+}, \otimes_{i}^{k}\right)$ and $R\left(\otimes^{-}, \otimes_{i}^{k}\right)$, there is

$$
\begin{aligned}
& R\left(\otimes^{+}, \otimes_{i}^{k}\right)=\sum_{j=1}^{n} w_{j} R\left(\otimes_{j}^{+}, \otimes_{i j}^{k}\right) \\
& R\left(\otimes^{-}, \otimes_{i}^{k}\right)=\sum_{j=1}^{n} w_{j} R\left(\otimes_{j}^{-}, \otimes_{i j}^{k}\right)
\end{aligned}
$$$$
R\left(\otimes^{+}, \otimes_{i}^{k}\right) \text { represents the grey correlation degree }
$$
between the evaluation value of scheme $x_{i}$ given by decision expert $e_{k}$ and the positive ideal scheme, $R\left(\otimes^{+}, \otimes_{i}^{k}\right)$ represents the grey correlation degree between it and the negative ideal scheme; $w_{j}$ is the attribute weight obtained by Step 2 ; both $R\left(\otimes_{j}^{+}, \otimes_{i j}^{k}\right)$ and $R\left(\otimes_{j}^{-}, \otimes_{i j}^{k}\right)$ is the grey correlation coefficient, and their expressions are:

$$
\begin{aligned}
& R\left(\otimes_{j}^{+}, \otimes_{i j}^{k}\right)=\frac{\min _{i} \min _{j} d\left(\otimes_{j}^{+}, \otimes_{i j}^{k}\right)+\xi \max _{i} \max _{j} d\left(\otimes_{j}^{+}, \otimes_{i j}^{k}\right)}{d\left(\otimes_{j}^{+}, \otimes_{i j}^{k}\right)+\xi \max _{i} \max _{j} d\left(\otimes_{j}^{+}, \otimes_{i j}^{k}\right)} \\
& R\left(\otimes_{j}^{-}, \otimes_{i j}^{k}\right)=\frac{\min _{i} \min _{j} d\left(\otimes_{j}^{-}, \otimes_{i j}^{k}\right)+\xi \max _{i} \max _{j} d\left(\otimes_{j}^{-}, \otimes_{i j}^{k}\right)}{d\left(\otimes_{j}^{-}, \otimes_{i j}^{k}\right)+\xi \max _{i} \max _{j} d\left(\otimes_{j}^{-}, \otimes_{i j}^{k}\right)}
\end{aligned}
$$

3) Apply Eq. (11) to calculate the closeness of the grey association of decision maker $e_{k}$ with respect to scheme $x_{i}$

$$
\delta_{i}^{k}=\frac{R\left(\otimes^{+}, \otimes_{i}^{k}\right)}{R\left(\otimes^{+}, \otimes_{i}^{k}\right)+R\left(\otimes^{-}, \otimes_{i}^{k}\right)}
$$

4) Calculate the closeness of the grey association of scheme $x_{i}$

$\delta_{i}=\sum_{k=1}^{s} \lambda_{k} \delta_{i}^{k}$

Among them, $\lambda_{k}(k=1,2, \ldots, s)$ are the expert weights obtained by Step 3 . 


\section{EXAMPLES}

For comparison, the cases pointed by Yue et al. are used for analysis $[14,15]$. At the end of the year, a certain university wants to evaluate the satisfaction of three leaders (Professor $f_{1}$, Associate Professor $f_{2}, f_{3}$ ). There are now three groups composed of the general public: teachers $d_{1}$, researchers $d_{2}$, and college students $d_{3}$ as decision makers. Including: leadership, fulfilment, working methods, the score is between 0 and 100 , the decision-maker's score for the evaluated object is expressed by the interval grey number, and the evaluation matrix given by each decisionmaker is as follows:

$$
\begin{aligned}
& A_{1}=\left[\begin{array}{lll}
{[60,90]} & {[72,86]} & {[85,92]} \\
{[77,81]} & {[69,93]} & {[83,88]} \\
{[80,96]} & {[59,87]} & {[68,85]}
\end{array}\right] \\
& A_{2}=\left[\begin{array}{lll}
{[77,83]} & {[68,86]} & {[82,90]} \\
{[92,98]} & {[76,86]} & {[65,87]} \\
{[79,85]} & {[72,92]} & {[81,97]}
\end{array}\right] \\
& A_{3}=\left[\begin{array}{lll}
{[85,96]} & {[76,86]} & {[80,97]} \\
{[79,87]} & {[75,89]} & {[81,93]} \\
{[62,82]} & {[84,89]} & {[78,82]}
\end{array}\right]
\end{aligned}
$$

Step 1 The expert preference matrix is normalized, and the standardized evaluation matrix of each decision maker is obtained as follows:

$$
R_{1}=\left[\begin{array}{lll}
{[0.6522,0.9783]} & {[0.7826,0.9348]} & {[0.9239,1.0000]} \\
{[0.8279,0.8710]} & {[0.7419,1.0000]} & {[0.8925,0.9462]} \\
{[0.8333,1.0000]} & {[0.6146,0.9063]} & {[0.7083,0.8854]}
\end{array}\right]
$$$$
R_{2}=\left[\begin{array}{lll}
{[0.8556,0.9222]} & {[0.7556,0.9556]} & {[0.9111,1.0000]} \\
{[0.9490,1.0000]} & {[0.7755,0.8776]} & {[0.6633,0.8776]} \\
{[0.8495,0.9140]} & {[0.7742,0.9892]} & {[0.8710,1.0000]}
\end{array}\right]
$$$$
R_{3}=\left[\begin{array}{lll}
{[0.8763,0.9897]} & {[0.7835,0.8866]} & {[0.8247,1.0000]} \\
{[0.8495,0.9355]} & {[0.8065,0.9570]} & {[0.8710,1.0000]} \\
{[0.6966,0.9213]} & {[0.9438,1.0000]} & {[0.8764,0.9213]}
\end{array}\right]
$$

Step 2 Determine attribute weight

According to the norm matrix $R_{1}, R_{2}, R_{3}$, the decision information matrix $\Delta_{1}$ of each scheme under attribute $u_{1}$ is obtained as:

$$
\Delta_{1}=\left[\begin{array}{lll}
{[0.6522,0.9783]} & {[0.8279,0.8710]} & {[0.8333,1.0000]} \\
{[0.8556,0.9222]} & {[0.9490,1.0000]} & {[0.8495,0.9140]} \\
{[0.8763,0.9897]} & {[0.8495,0.9355]} & {[0.6966,0.9213]}
\end{array}\right]
$$

$$
=\left[\begin{array}{l}
\otimes_{1}^{1} \\
\otimes_{1}^{2} \\
\otimes_{1}^{3}
\end{array}\right]
$$

Apply Eq. (4) to calculate the grey correlation between each two row vectors, then there is $\left(\otimes_{1}^{1}, \otimes_{1}^{2}\right)=$ $0.7781, R\left(\otimes_{1}^{1}, \otimes_{1}^{3}\right)=0.9509, R\left(\otimes_{1}^{2}, \otimes_{1}^{3}\right)=0.8502$.

The cumulative grey correlation degree is $E_{1}=2.5792$, and similarly, the cumulative grey correlation degrees of the evaluation indicators $u_{2}, u_{3}$ are calculated as $E_{2}=$
2.3129 and $E_{3}=2.2701$, respectively. Apply Eq. (6) to get the attribute weight vector as $=[0.3076,0.3430,0.3494]$.

Step 3 Determine expert weights. Calculate the relevance of each two experts, respectively: $\left(\otimes_{1}, \otimes_{2}\right)=0.7255, R\left(\otimes_{1}, \otimes_{3}\right)=0.7174, R\left(\otimes_{2}, \otimes_{3}\right)=$ 0.7940 .

Then there is:

For expert $e_{1}$, there is $E_{1}=R\left(\otimes_{1}, \otimes_{2}\right)+R\left(\otimes_{1}, \otimes_{3}\right)=1.4429$.

For expert $e_{2}$, there is $E_{2}=R\left(\otimes_{1}, \otimes_{2}\right)+R\left(\otimes_{2}, \otimes_{3}\right)=1.5195$.

For expert $e_{3}$, there is $E_{3}=R\left(\otimes_{1}, \otimes_{3}\right)+R\left(\otimes_{2}, \otimes_{3}\right)=1.5114$

The expert weights for solving are: $\lambda=(0.3443$, $0.3270,0.3287)$.

Step 4 Calculate the grey closeness of each scheme

According to the norm matrix $R_{1}, R_{2}, R_{3}$, the positive ideal scheme $\otimes^{+}$and the negative ideal scheme $\otimes^{-}$are obtained as

$$
\begin{aligned}
& \otimes^{+}=([0.9490,1.0000] \quad[0.9438,1.0000] \quad[0.9239,1.0000]) \\
& \otimes^{-}=([0.6522,0.8710] \quad[0.6146,0.8776] \quad[0.6633,0.8776])
\end{aligned}
$$

Combined with attribute weight $w$, there are:

$$
\begin{aligned}
& R\left(\otimes^{+}, \otimes_{1}^{1}\right)=0.7262, R\left(\otimes^{-}, \otimes_{1}^{1}\right)=0.5840 \Rightarrow \delta_{1}^{1}=0.5543 \\
& R\left(\otimes^{+}, \otimes_{2}^{1}\right)=0.5676, R\left(\otimes^{-}, \otimes_{2}^{1}\right)=0.5190 \Rightarrow \delta_{2}^{1}=0.5224 \\
& R\left(\otimes^{+}, \otimes_{3}^{1}\right)=0.5752, R\left(\otimes^{-}, \otimes_{3}^{1}\right)=0.8053 \Rightarrow \delta_{3}^{1}=0.4167 \\
& R\left(\otimes^{+}, \otimes_{1}^{2}\right)=0.6136, R\left(\otimes^{-}, \otimes_{1}^{2}\right)=0.4960 \Rightarrow \delta_{1}^{2}=0.5530 \\
& R\left(\otimes^{+}, \otimes_{2}^{2}\right)=0.5653, R\left(\otimes^{-}, \otimes_{2}^{2}\right)=0.6720 \Rightarrow \delta_{2}^{2}=0.4569 \\
& R\left(\otimes^{+}, \otimes_{3}^{2}\right)=0.5836, R\left(\otimes^{-}, \otimes_{3}^{2}\right)=0.5053 \Rightarrow \delta_{3}^{2}=0.5360 \\
& R\left(\otimes^{+}, \otimes_{1}^{3}\right)=0.5475, R\left(\otimes^{-}, \otimes_{1}^{3}\right)=0.6517 \Rightarrow \delta_{1}^{3}=0.4566 \\
& R\left(\otimes^{+}, \otimes_{2}^{3}\right)=0.5674, R\left(\otimes^{-}, \otimes_{2}^{3}\right)=0.6053 \Rightarrow \delta_{2}^{3}=0.4838 \\
& R\left(\otimes^{+}, \otimes_{3}^{3}\right)=0.6291, R\left(\otimes^{-}, \otimes_{3}^{3}\right)=0.6459 \Rightarrow \delta_{3}^{3}=0.4934
\end{aligned}
$$

Finally, combined with expert weight $\lambda$, the group decision results are: $\delta_{1}=0.5217, \delta_{2}=0.4883, \delta_{3}=0.4809$, respectively. According to this, the rankings of the obtained schemes are: $f_{1} \succ f_{2} \succ f_{3}$ and the professor's satisfaction is the highest.

1) Comparative analysis of expert weights

Using this method and Yue et al. studies to get the weight of decision makers is shown in Tab. $1[14,15]$.

It can be seen from Tab. 1: The ranking of the weights of experts from Yue et al. studies is the same. The ranking of the importance of teachers, researchers and college students in this ranking is: $d_{3} \succ d_{2} \succ d_{1}$; the ranking of them in this article is: $d_{1} \succ d_{3} \succ d_{2}$. The reason is that the idea of solving expert weights is different. The method in this paper is based on the degree of correlation between the information of experts. The studies are based on the correlation between expert decision information and group decision information (or ideal scheme) [14, 15]. In practice, the satisfaction of professors $f_{1}$ and associate 
professors $f_{2}, f_{3}$ is evaluated in terms of leadership, fulfillment, and working methods. The decision information given by teachers is more persuasive than that of researchers $d_{2}$ and college students $d_{3}$, and the corresponding weight should be higher.

Table 1 Decision maker weights
\begin{tabular}{|c|c|c|c|}
\hline & $\lambda_{1}$ & $\lambda_{2}$ & $\lambda_{3}$ \\
\hline This article & 0.3443 & 0.3270 & 0.3287 \\
\hline Yue [14] & 0.3330 & 0.33353 & 0.33347 \\
\hline Yan et al. [15] & 0.3006 & 0.3386 & 0.3608 \\
\hline
\end{tabular}

Note: $\lambda_{1}, \lambda_{2}, \lambda_{3}$ in the table represents the weight of experts corresponding to teachers $d_{1}$, researchers $d_{2}$ and college students $d_{3}$.

Table 2 Group decision results and ranking

\begin{tabular}{|c|c|c|c|c|c|c|}
\hline & \multicolumn{2}{|c|}{ This article } & \multicolumn{2}{|c|}{ Yue [14] } & \multicolumn{2}{|c|}{ Yan et al. [15] } \\
\hline & Evaluation & ranking & Evaluation & ranking & Evaluation & ranking \\
\hline$f_{1}$ & 0.5217 & 1 & 1.72505 & 3 & 1.0387 & 1 \\
\hline$f_{2}$ & 0.4883 & 2 & 1.72780 & 1 & 0.9949 & 3 \\
\hline$f_{3}$ & 0.4809 & 3 & 1.72746 & 2 & 1.0306 & 2 \\
\hline
\end{tabular}

Note: In the original reference [15], the group decision results are compared in the form of interval grey numbers, but Yan et al. [17] pointed out that the traditional interval grey number comparison has defects and needs to convert the interval grey number to a relative kernel.

2) Comparative analysis of decision results

Using the method of this paper and Yue et al. studies to get the evaluation value and ranking result of the scheme, see Tab. $2[14,15]$.

It can be seen from Tab. 2 that the method, Yue et al. gives the pros and cons of the schemes as follows: $f_{1} \succ f_{2} \succ f_{3}, f_{2} \succ f_{3} \succ f_{1}, f_{1} \succ f_{3} \succ f_{2}$ respectively [14,15]. Among them: 1) Both the article and the Yan's study [15] believe that the professor's satisfaction is the highest, which is the same as that of the professor when the original literature information was collected; 2) For the associate professors and associate professors, both the article and the Yue's study [14] believe that. In summary, the method in this paper is more effective than the studies $[14,15]$. This is because the method in this paper can comprehensively consider the relationship between each program and the positive and negative ideal programs, so that the decision will not produce deviations, that is, there will be no inconsistencies in changes; the studies about Yue and Yan et al. use weighting Method calculation, the decision result is one-sided $[14,15]$.

Compared with the above two methods, the advantages of this article are: 1) The determination of attribute weights and expert weights is based on the comparison and analysis of the original data, without interference of other factors; 2) The comprehensive consideration of the relationship between each program and the positive and negative ideal programs is more realistic. Therefore, the model solution results are more scientific and reasonable.

\section{CONCLUSIONS}

Aiming at the problem of interval grey number multiattribute group decision making with completely unknown expert weights and attribute weights, an interval grey number type group decision method based on the closeness of grey association is proposed. On the one hand, the grey correlation model based on the degree of separation is used to analyse the correlation between decision information to obtain attribute weights and expert weights; on the other hand, the grey correlation closeness model is used to comprehensively consider each program and the positive and negative ideal programs so that there is no deviation in decision-making. The comparison with other methods verifies that this method is more reasonable.

The proposed method is based on the expected utility theory. However, in the actual decision-making process, the psychological behaviour of decision makers does not always pursue utility maximization, but is characterized by reference dependence and loss avoidance. Therefore, the next step is to study the interval grey number multiattribute decision-making method considering the psychological behaviour of decision makers.

\section{Acknowledgment}

This project was funded by the Freight Mobility Research Institute (FMRI), one of the TIER 1 Transportation Centers that were selected by the Office of the Assistant Secretary for Research and Technology (OST-R), U.S. Department of Transportation (US DOT). The paper was also based on a research project financed by the National Natural Science Foundation of China (Grant No. 61806021, 71701022), the Humanities and Social Sciences Project of Education Ministry (Grant No.15YJCZH093), the Social Science Fund Project in Shaanxi Province (Grant No.2014P08), Basic Scientific Research Funds of Central Universities (Grant No. 300102239632, 300102238401).

\section{REFERENCES}

[1] Badi, I. \& Pamucar, D. (2020). Supplier selection for steelmaking company by using combined Grey-MARCOS methods. Decision Making: Applications in Management and Engineering, 3(2), 37-48. https://doi.org/10.31181/dmame2003037b

[2] Si, A., Das, S., \& Kar, S. (2019). An approach to rank picture fuzzy numbers for decision making problems. Decision Making: Applications in Management and Engineering, 2(2), 54-64. https://doi.org/10.31181/dmame1902049s

[3] Xu, Z. S. (2004). Uncertain Multiple Attribute Decision Making Methods and Application, Beijing: Tsinghua University Press.

[4] Wang, X. \& Dang, Y. G. (2015). Approach for multiple attribute decision-making with interval grey number based on choquet integral. Systems Engineering and Electronics, 37(5), 1106-1110.

[5] Guo, S. D., Liu, S. F., \& Fang, Z. G. (2016). Multi-attribute decision making model based on the kernel and the degree of greyness of the interval grey numbers. Control and Decision, 31(6), 1042-1046.

[6] Teki, S. M., Banothu, B., \& Varma, M. K. (2019). An unrealized algorithm for effective privacy preservation using classification and regression trees. Revue d'Intelligence Artificielle, 33(4), 313-319. https://doi.org/10.18280/ria.330408 
[7] Zhang, S. F. (2019). Classification of urban land use based on graph theory and geographic information system. Ingénierie des Systèmesd'Information, 24(6), 633-639. https://doi.org/10.18280/isi.240611

[8] Zhu, P., Wang, X., Jia, D., Guo, Y., Li, S., \& Chu, C. (2020). Investigating the co-evolution of node reputation and edgestrategy in prisoner's dilemma game. Applied Mathematics and Computation, 386, 125474. https://doi.org/10.1016/j.amc.2020.125474

[9] Jiang, S. Q., Liu, S. F., Liu, Z. X., \& Fang, Z. G. (2017). The incidence consistence decision model of interval grey number based on the information decomposition. Control and Decision, 32(1), 111-116.

[10] Liu, Z. X., Liu, S. F., \& Fang, Z. G. (2017). Decision making model of grey comprehensive correlation and relative close degree based on kernel and greyness degree. Control and Decision, 32(8), 1475-1480.

[11] Yang, B. H., Fang, Z. G., Zhou, W., \& Liu, J. (2012). Incidence decision model of multi-attribute interval grey number based on information reduction operator. Control and Decision, 27(2), 182-186.

[12] Zeng, B., Liu, S. F., Li, C., \& Chen, J. M. (2013). Grey target decision-model of interval grey number based on cobweb area. Systems Engineering and Electronics, 35(11), 23292334.

[13] Chen, X. X. \& Liu, S. F. (2008). Study on grey multiple attribute group decision-making method without weight information. Chinese Journal of Management Science, 16(5), 146-152.

[14] Yue, Z. L. (2012). Application of the projection method to determine weights of decision makers for group decision making. Scientia Iranian, 19(3), 872-878. https://doi.org/10.1016/j.scient.2012.03.008

[15] Yan, S. L., Liu, S. F., Fang, Z. G., \& Wu, L. F. (2014). Method of determining weights of decision makers and attributes for group decision making with interval grey numbers. Systems Engineering - Theory \& Practice, 34(9), 2372-2378

[16] Li, Y. L., Yin, X. L., \& Yang, J. (2017). Multi-attribute group decision making model based on kernel and degree of greyness of interval grey numbers. Fire Control \& Command Control, 42(3), 17-20.

[17] Yan, S. L., Liu, S. F., Zhu, J. J., \& Fang, Z. G. (2014). The ranking method of grey numbers based on relative kernel and degree of accuracy. Control and Decision, 29(2), 315-319.

\section{Contact information:}

\section{Dan LIU}

1) School of Economics and Management, Chang'an University,

Xi'an 710064, China

2) Freight Mobility Research Institute, Department of Civil,

Environmental, and Geomatics Engineering, Florida Atlantic University,

Boca Raton, FL, 33431, USA

E-mail: dan_liuedu@sohu.com

\section{Bin HU}

(Corresponding author)

School of Automation, Northwestern Polytechnical University,

Xi'an 710064, China

E-mail: BinHu201205@hotmail.com

\section{Zhou DING}

School of Automation, Northwestern Polytechnical University,

Xi'an 710064, China

E-mail: dingdaisy@163.com

\section{Evangelos I. KAISAR}

Freight Mobility Research Institute, Department of Civil,

Environmental, and Geomatics Engineering, Florida Atlantic University,

Boca Raton, FL, 33431, USA

E-mail: ekaisar@fau.edu 